\title{
DYNAMICS OF A REINFORCED VISCOELASTIC PLATE
}

\author{
IGOR V. ANDRIANOV, JAN AWREJCEWICZ, AND IRINA V. PASICHNIK
}

Received 22 December 2005; Accepted 2 February 2006

Oscillations and static bending deformation of a viscoelastic reinforced plate are considered. Analytical solutions are derived. An asymptotic technique, based on the homogenization method, is used for this purpose. In addition, a special perturbation approach is employed. An example is given for the purpose of illustration. The approximate analytical expressions are shown to adequately meet the requirements of optimal structural design.

Copyright (c) 2006 Igor V. Andrianov et al. This is an open access article distributed under the Creative Commons Attribution License, which permits unrestricted use, distribution, and reproduction in any medium, provided the original work is properly cited.

\section{Introduction}

Reinforced plates and shells are described by partial differential equations with rapidly varying coefficients, and their stress-strain state may be represented as a sum of a slowand a fast-varying components $[2,3,11]$. In many physical problems, some variables may vary rather slowly, while others change fast. It is natural to ask whether it would be appropriate first to study the overall structure at hand, neglecting its local distinctive features, and next to investigate the system locally.

The paper is structured as follows. Section 2 presents the governing relationships. Section 3 deals with the homogenization procedure in general. Solutions for the local problem and the boundary layer are given in Section 4. Finally, a discussion and comments regarding the results obtained are given in Section 5.

\section{Governing relationships and estimates}

The derivation of the equilibrium motion equations for reinforced plates and shells taking into account discrete arrangement of ribs is the subject of numerous studies [1,8-10, 12]. One can conclude on the basis of the corresponding results that a $3 \mathrm{D}$ theory of elasticity is needed for the correct description of the plate behavior in the vicinity of the rib. Out of these narrow regions, the results obtained for different contact hypotheses coincide if 
the width of the rib is not large compared to the thickness of the plate. Therefore, the line contact approximation will be explored further. The ribs themselves are treated in the framework of the Kirchhoff-Klebsch hypotheses. Viscoelasticity according to [4-6] is taken into account.

Consider oscillations of a rectangular plate $\left(0 \leq x \leq L_{1},-L_{2} \leq y \leq L_{2}\right)$, supported by a regular array of $N=2 n+1$ ribs. The stiffness extended along the $x$-direction. Each rib is symmetric with respect to the middle surface of the plate. Materials of plate and ribs are linear viscoelastic with instantaneous Young modulus $E$ and Poisson coefficient $\nu$. The governing equation of motion may be written as follows:

$$
\bar{D} \nabla^{4} \bar{W}+\bar{E}_{1} I \Phi(y) \bar{W}_{x x x x}+[r+R \Phi(y)] \bar{W}_{t t}=0,
$$

where

$$
\begin{gathered}
\Phi(y) \sum_{i=-0.5(N-1)}^{i=0.5(N-1)} \delta(y-i b), \quad b=\frac{2 L_{2}}{N+1}, \quad \bar{D}=D \Gamma, \quad \overline{E_{1} I}=E_{1} I \Gamma, \\
\Gamma(\bar{W})=\bar{W}+-\int_{-\infty}^{t} G(t-\tau) \bar{W}(\tau) d \tau, \quad D=\frac{E h^{3}}{12\left(1-\nu^{2}\right)},
\end{gathered}
$$

and $t$ is the time, $\bar{W}(x, y, t)$ is the normal displacement, $E_{1}$ is the rib Young modulus, $h$ is the plate thickness, $r, R$ are the plate and rib material density, $I$ is the moment of rib cross-section, $\delta(x)$ is Dirac delta-function, $G(t-\tau)$ is the kernel of relaxation velocity.

The boundary conditions, without loss of generality, can be written in the form

$$
\begin{array}{ll}
\bar{W}=\bar{W}_{y}=0 \quad \text { for } y= \pm L_{2}, & \\
\bar{W}=\bar{W}_{x x}=0 & \text { for } x=0, L_{1}, \quad \text { or } \\
\bar{W}=\bar{W}_{x}=0 & \text { for } x=0, L_{1} .
\end{array}
$$

The conditions of continuity and equilibrium are

$$
\begin{gathered}
\bar{W}^{+}=\bar{W}^{-}=\bar{W}, \quad \bar{W}_{y}^{+}=\bar{W}_{y}^{-}, \quad \bar{W}_{y y}^{+}=\bar{W}_{y y}^{-}, \\
\bar{D}\left(\bar{W}_{y y y}^{+}-\bar{W}_{y y y}^{-}\right)=\bar{E}_{1} I W_{x x x x}+R \bar{W}_{t t},
\end{gathered}
$$

where $(\cdots)^{( \pm)}=\lim _{y \rightarrow i b \pm 0}(\cdots)$.

The torsion rigidity of the ribs is neglected for the thin stiffness.

Because of the discontinuities in the coefficient of (2.1), one should find its solution in the framework of the distribution theory [7]. Namely, the solutionis defined as 
distribution $w$ satisfying the integral identity

$$
\begin{gathered}
{[w, z]+\int_{-L 2}^{L 2} \int_{0}^{L 1}[r+R \phi(y)] \bar{W}_{t t} z d x d y=0} \\
{[w, z]+\int_{-L 2}^{L 2} \int_{0}^{L 1}\left\{\left[\bar{D}+E_{1} I \phi(y)\right] \frac{\partial^{2} \bar{W} \partial^{2} Z}{\partial x^{2} \partial x^{2}}+2 \frac{\partial^{2} \bar{W}}{\partial x \partial y} \frac{\partial^{2} Z}{\partial x \partial y}+\frac{\partial^{2} \bar{W} \partial^{2} Z}{\partial y^{2} \partial y^{2}}\right\} d x d y,}
\end{gathered}
$$

where $z(x, y)$ is smooth function satisfying conditions (2.3), (2.4) or (2.3), (2.5).

The problems (2.7), (2.3), (2.4) or ((2.7), (2.3), (2.5)) have a countable real spectrum $\left\{\lambda_{k}\right\}(k=1,2, \ldots)$. The corresponding eigenfunctions $w_{k}$ are also real and form orthogonal basis in sense of a scalar product $[w, z]$.

The following Ansatz is used:

$$
\bar{W}=w(x, y) \exp (i \Lambda t)
$$

where $\Lambda=\omega+i a, \omega$ is the frequency and $a$ is the damping factor, and the following relation is applied:

$$
\int_{-\infty}^{t} G(t-\tau) e^{i \Lambda t} d \tau=e^{i \Lambda t} C
$$

where $C=\int_{0}^{t} G(\theta) e^{i \Lambda t} d \theta$.

Then (2.1) and conditions (2.6) may be reduced to the following dimensionless form:

$$
\begin{aligned}
& \nabla^{4} W+\alpha \phi(\varphi) W_{\xi \xi \xi \xi}-[1-\lambda \rho \phi(\varphi)] W=0, \\
& W^{+}=W^{-}=W, \quad W_{\eta_{1} \eta_{1}}^{+}=W_{\eta_{1} \eta_{1}}^{-}, \\
& -W_{\eta_{1} \eta_{1} \eta_{1}}^{+}+W^{-} \eta_{1} \eta_{1} \eta_{1}=\varepsilon\left(\alpha \frac{\partial^{4}}{\partial \xi^{4}}-\lambda \rho\right) W
\end{aligned}
$$

where

$$
\begin{gathered}
\nabla^{4}=\frac{\partial^{4}}{\partial \xi^{4}}+2 \frac{\partial^{4}}{\partial \xi^{2} \partial \eta_{1}^{2}}+\frac{\partial^{4}}{\partial \eta_{1}^{4}}, \quad \xi=\frac{x}{2 L_{2}}, \quad \eta_{1}=\frac{y}{2 L_{2}}, \\
\alpha=\frac{E_{1} I}{D b}, \quad \varphi=\frac{y}{b}, \quad \rho=\frac{R}{r b}, \quad \phi(\varphi)=\sum_{i=-0.5(N-1)}^{i=0.5(N-1)} \delta(\varphi-1), \\
\lambda=\frac{16 r}{D(1-C)} \Lambda^{2} L_{2}^{4}, \quad(\cdots)^{( \pm)}=\lim _{\varphi \rightarrow-( \pm) 0}(\cdots), \quad \varepsilon=\frac{b}{2 L_{2}} .
\end{gathered}
$$

For a real reinforced plates $\varepsilon \ll 1, \alpha$ and $\rho$ are of the order of $\varepsilon^{-1}$.

The conventional approaches for reinforced plates are efficient in two opposite limiting cases. A large number of ribs are the firstlimit. This limit is analyzed using the structurally 
orthotropic theory (SOT). A small number of ribs are the second limit. The corresponding technique is based on the separation of plate into elastic panels between the ribs in the complex with proper compatibility conditions on the rib lines. However, the case of a finite number of ribs is extremely important for applications. SOT can be correctly used for estimation in the low-frequency region of frequencies and displacements. At the same time, the application of SOT is not correct for transverse shear forces and the description of bending moments. Unfortunately, exploring the technique corresponding to the small number of ribs limit is not efficient here. To overcome these difficulties, a homogenization method is used.

\section{Homogenization procedure}

An explanation of the problem stated above is important for both theoretical and computational considerations. Due to the complexity of its structure, any kind of calculation is difficult to perform for a reinforced plate. An approximation of the problem at hand by a "homogenized" one is therefore desirable. The method used here is a variant of the multiscaling technique. It is well known that this is a general method applicable to a wide range of problems. The problems are characterized by having two physical processes, each with its own scales, and with the two processes acting simultaneously. "Slow" $\left(\eta=\eta_{1}\right)$ and "fast" $(\varphi)$ variables will be used. Then derivative $\partial / \partial \eta_{1}$ has the form

$$
\frac{\partial}{\partial \eta_{1}}=\frac{\partial}{\partial \eta}+\varepsilon^{-1} \frac{\partial}{\partial \varphi} .
$$

The solution of boundary value problem (2.10) is represented in the form of a formal expansion

$$
\begin{gathered}
W(x, y)=\left[W_{00}(\xi, \eta)+\varepsilon W_{01}(\xi, \eta)+\varepsilon^{2} W_{02}(\xi, \eta)+\cdots\right] \\
+\varepsilon^{3}\left[W_{1}(\xi, \eta, \varphi)+\varepsilon W_{2}(\xi, \eta, \varphi)+\cdots\right], \\
\lambda=\varepsilon^{-1} \lambda_{0}+\lambda_{1}+\varepsilon \lambda+\cdots .
\end{gathered}
$$

It is assumed that $W_{j}(x, \eta, \varphi+1)=W_{j}(x, \eta, \varphi), j=1,2, \ldots$

Substituting series (3.2) into boundary value problem (2.10), taking into account relation (3.1), and splitting it with respect to powers of $\varepsilon$, one obtains a recurrent sequence 
of boundary value problems

$$
\begin{aligned}
& W_{1 \varnothing \varnothing \varnothing \varnothing}-\Pi_{0} W_{00}=0 \text {, } \\
& W_{2 \varnothing \varnothing \varnothing \varnothing}+\Pi_{0} W_{01}=\Pi_{1} W_{1}-\nabla^{4} W_{00}, \\
& W_{3 \varnothing \varnothing \varnothing \varnothing}+\Pi_{0} W_{02}=\Pi_{1} W_{2}+\Pi_{2} W_{1}-\nabla^{4} W_{01}, \\
& W_{4 \varnothing \varnothing \varnothing \varnothing}+\Pi_{0} W_{03}=\Pi_{1} W_{3}+\Pi_{2} W_{2}+\Pi_{3} W_{1}-\nabla^{4} W_{02} \text {, } \\
& W_{5 \varnothing \varnothing \varnothing \varnothing}+\Pi_{0} W_{04}=\sum_{i=1}^{3} \Pi_{i} W_{5-1}-\nabla^{4}\left(W_{03}+W_{1}\right) \\
& W_{k \varnothing \varnothing \varnothing \varnothing}+\Pi_{0} W_{0 k-1}=\sum_{i=1}^{3} \Pi_{i} W_{k-1}+\nabla^{4}\left(W_{0 k-2}+W_{k-4}\right), \\
& \Pi_{0}=\alpha \phi \frac{\partial^{4}}{\partial \xi^{4}}-\lambda_{0}[1-\rho \phi(\varphi)], \quad \Pi_{1}=-4 \frac{\partial^{4}}{\partial \varphi^{3} \partial \eta} \\
& \Pi_{2}=-6 \frac{\partial^{4}}{\partial \varphi^{2} \partial \eta^{2}}-2 \frac{\partial^{4}}{\partial \varphi^{2} \partial \xi^{2}}, \quad \Pi_{3}=\Pi_{31}+\Pi_{30}=-\left(\frac{\partial^{4}}{\partial \varphi \partial \eta \partial \xi^{2}}+\frac{\partial^{4}}{\partial \varphi \partial \eta^{3}}\right)-\alpha \phi \frac{\partial^{4}}{\partial \xi^{4}}
\end{aligned}
$$

Upon splitting, the boundary conditions take the following form:

$$
\begin{gathered}
\text { for } \xi=0, \quad d: W_{0 i}=-W_{i-2}, \quad W_{0 i \xi \xi}=-W_{i-2 \xi \xi}, \\
\text { or } W_{01}=-W_{i-2}, \quad W_{0 i \xi}=-W_{i-2 \xi}, \\
\text { for } \eta= \pm 0.5: W_{0 i}=-W_{i-2}, \quad W_{0 i \eta}=-W_{i-1 \varphi}-W_{i-2 \eta} .
\end{gathered}
$$

Here $i=0,1, \ldots ; W_{i}=0$ for $i \leq 0 ; d=L_{1} / 2 L_{2}$.

Consider the following homogenization operator:

$$
(\widehat{\cdots})=\frac{1}{N+1} \int_{-0.5(N+1)}^{0.5(N+1)}(\cdots) d \varphi .
$$

One must take into account that $\widehat{W}_{0 i}=W_{0 i}, \widehat{\phi}=1, \Pi_{1} \widehat{W}_{i}=\Pi_{2} \widehat{W}_{i}=\Pi_{31} \widehat{W}_{i}=0$. 
The following is easily obtained from (3.3)-(3.6) by applying the homogenization operator defined by (3.7)

$$
\begin{gathered}
\Pi_{00} W_{00}=\left[\alpha \frac{\partial^{4}}{\partial \xi^{4}}-\lambda_{0}(1+\rho)\right] W_{00}=0, \\
\Pi_{00} W_{01}=-\nabla^{4} W_{00}, \\
\Pi_{00} W_{02}=-\nabla^{4} W_{00}, \\
\Pi_{00} W_{03}=\Pi_{30} \widehat{W}_{1} \nabla^{4} W_{02}, \\
\vdots \\
\Pi_{00} W_{0 k}=\Pi_{30} \widehat{W}_{k-2}-\nabla^{4}\left(W_{0 k-1}+\widehat{W}_{k-3}\right), \\
\text { for } \xi=0, \quad \begin{array}{c}
d: W_{0 i}=-\widehat{W}_{i-2}, \quad W_{0 i \xi \xi}=-\widehat{W}_{i-2 \xi \xi} \\
\text { or } W_{01}=-\widehat{W}_{i-2}, \quad W_{0 i \xi}=-\widehat{W}_{i-2 \xi},
\end{array} \\
\text { for } \eta= \pm 0.5: W_{0 i}=-\widehat{W}_{0 i-2}, \quad W_{0 i \eta}=-\widehat{W}_{i-2 \eta},
\end{gathered}
$$

where $i=0,1, \ldots ; \widehat{W}_{i}=0$ for $i \leq 0$.

Equations (3.8) and (3.9) are combined to yield

$$
D_{1} W_{0 x x x x}+2 D W_{0 x x y y}+D W_{0 y y y y}-\Lambda_{1} W_{0}=0 .
$$

Here $D_{1}=D+E_{1} I / b ; \Lambda_{1}=\Lambda^{2}(r+R / b)$

$$
D_{1} W_{0 x x x x}+2 D W_{0 x x y y}+D W_{0 y y y y}-\Lambda_{1} W_{0}=0,
$$

where $D_{1}=D+E_{1} I / b ; \Lambda_{1}=\Lambda^{2}(r+R / b)$.

\section{Local solution}

Using boundary value problems (3.8)-(3.17), one can obtain a homogenized ("global") solution. However, it is very important to calculate the local component $W_{i}(i \geq 1)$ of the initial solution of the problem as well. Following boundary value problems, for the 
functions $W_{i}$,

$$
\begin{gathered}
W_{1 \varphi \varphi \varphi \varphi}=\Pi_{01} W_{00}=\left[\alpha \frac{\partial^{4}}{\partial \xi^{4}}-\lambda_{0} \rho\right] W_{00}, \\
W_{2 \varphi \varphi \varphi \varphi}=\Pi_{1} W_{1}-\Pi_{01} W_{01}, \\
\vdots \\
W_{k \varphi \varphi \varphi \varphi}=\sum_{i=1}^{3} \Pi_{i} \bar{W}_{k-i}-\nabla^{4} \bar{W}_{k-4}+\Pi_{01} W_{0 k-1}, \\
\vdots \\
\text { for } \varphi= \pm k \quad(k=0,1, \ldots, 0.5(N+1)) \quad W_{i}=W_{i \varphi}=0,
\end{gathered}
$$

exists where $(\cdots)=(\cdots)-(\widehat{\cdots})$.

The conditions of compatibility are automatically satisfied. Using (4.1) and boundary conditions (4.6), one obtains

$$
W_{1}=\frac{1}{24} \Pi_{01} W_{0} F_{4}(\varphi),
$$

where $F_{4}(\varphi)$ is a periodical function, $F_{4}(\varphi)=\varphi^{2}(\varphi-1)^{2}$ for $0 \leq \varphi \leq 1$.

For the governing variables,

$$
W_{1}=\frac{1}{24}\left(\frac{E_{1} I}{b} \frac{\partial^{4}}{\partial x^{4}}-\frac{R}{b} \Lambda^{2}\right) W_{0} y^{2}(y-b)^{2} .
$$

The expression for $W_{i}$ for $i>1$ may be written as follows:

$$
W_{1}=\frac{1}{24} \Pi_{01} W_{0 i-1} F_{4}(\varphi)+\sum_{j=0}^{3} c_{j}^{(i)} \varphi^{j}+\iiint \int\left[\sum_{j=1}^{3} \Pi_{j} \bar{W}_{i-j}-\nabla^{4} W_{i-4}\right] d \varphi d \varphi d \varphi d \varphi .
$$

The functions $c_{j}^{(i)}(\xi, \eta)$ satisfy boundary conditions (4.6)

\section{Concluding remarks}

A new approximate solution of the viscoelastic problem for a reinforced plate has been developed. The homogenization procedure and the averaging approach have made it possible to obtain an analytical form. The method can also be advantageously used for analysis of viscoelastic shells with periodic structures.

It is very important that obtained solution takes into account the real arrangement of the ribs.

Some problems in the theory of plates and shells, for which solutions were found at an initial level, are closed in certain aspects to those proposed above. However, a number of difficulties arise when studying reinforced plated and shells which cannot be overcome at 
the "intuitive level." These relate especially to dynamic and nonlinear problems with realistic boundary conditions. It is also not clear a priori which terms in the initial equations have to remain during the subsequent simplification. These difficulties can be overcome if we construct a grounded asymptotic procedure only. So, the above proposed solution is important from the engineering standpoint.

\section{References}

[1] I. Ya. Amiro and V. A. Zarutsky, Taking the discrete rib spacing into account when studying the stress-strained state, vibration and stability of ribbed shells (Review), International Applied Mechanics 34 (1998), no. 4, 299-314.

[2] I. V. Andrianov, V. A. Lesnichaya, and L. I. Manevitch, Homogenization Methods in Static and Dynamics of Reinforced Shells, Nauka, Moscow, 1985.

[3] J. Awrejcewicz, I. V. Andrianov, and L. I. Manevitch, Asymptotic Approaches in Nonlinear Dynamics. New Trends and Applications, Springer Series in Synergetics, Springer, Berlin, 1998.

[4] V. V. Bolotin, The Dynamical Stability of Elastic Systems, Holden-Day, California, 1964.

[5] M. G. Botogova and G. I. Mikhasev, Free vibrations of viscoelastic noncircular cylindrical shell under action of uniform axial loading, International Applied Mechanics 35 (1999), no. 11, 6874.

[6] R. M. Christensen, Introduction to the Viscoelastic Theory, Academic Press, New York, 1971.

[7] W. Kecs and P. P. Teodorescu, Applications of the Theory of Distributions in Mechanics, Editura Academiei Române, Bucharest; Abacus Press, Tunbridge Wells, 1974.

[8] R. V. Kohn and M. Vogelius, A new model for thin plates with rapidly varying thickness, International Journal of Solids and Structures 20 (1984), no. 4, 333-350.

[9] _ A new model for thin plates with rapidly varying thickness. III. Comparison of different scalings, Quarterly of Applied Mathematics 44 (1986), no. 1, 35-48.

[10] T. Lewiński and J. J. Telega, Plates, Laminates and Shells. Asymptotic Analysis and Homogenization, Series on Advances in Mathematics for Applied Sciences, vol. 52, World Scientific, New Jersey, 2000.

[11] L. I. Manevitch, I. V. Andrianov, and V. G. Oshmyan, Mechanics of Periodically Heterogeneous Structures, Foundations of Engineering Mechanics, Springer, Berlin, 2002.

[12] P. A. Zhilin, Linear theory of ribbed shells, Mechanics of Solids 5 (1970), no. 6, 150-162, in Russian.

Igor V. Andrianov: Institut für Allgemeine Mechanikder RWTH Aachen, Templergraben 64, 52062 Aachen, Germany

E-mail address: igor_andrianov@hotmail.com

Jan Awrejcewicz: Department of Automatics and Biomechanics, Technical University of Lódź, 1/15 Stefanowski Street, 90-924 Lódź, Poland

E-mail address: awrejcew@p.lodz.pl

Irina V. Pasichnik: Department of Higher Mathematics, National Metallurgical Academy of Ukraine, Gagarina 23, Dnipropetrovsk 49005, Ukraine

E-mail address: anatoliy@kpm.dp.ua 


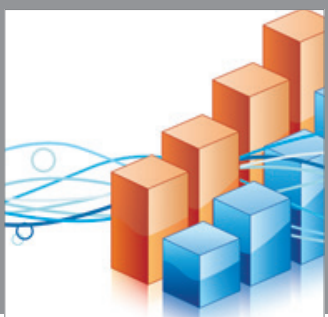

Advances in

Operations Research

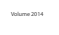

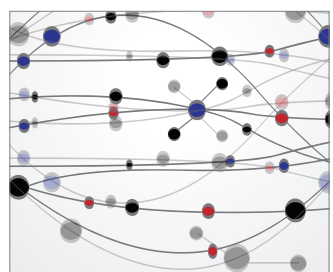

\section{The Scientific} World Journal
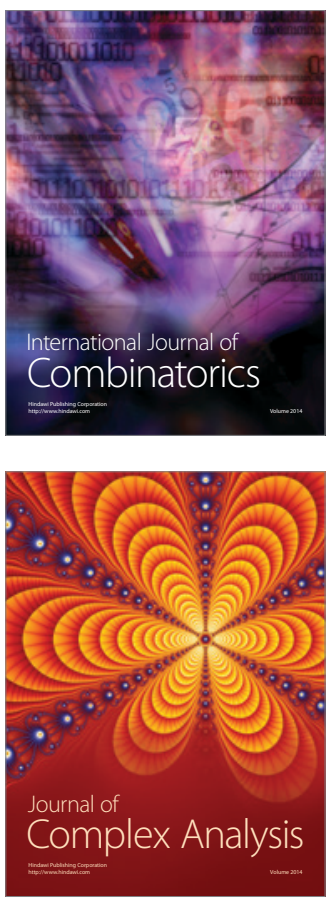

International Journal of

Mathematics and

Mathematical

Sciences
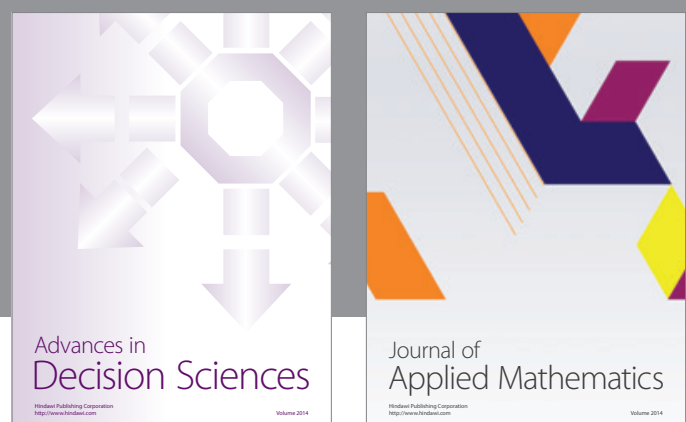

Journal of

Applied Mathematics
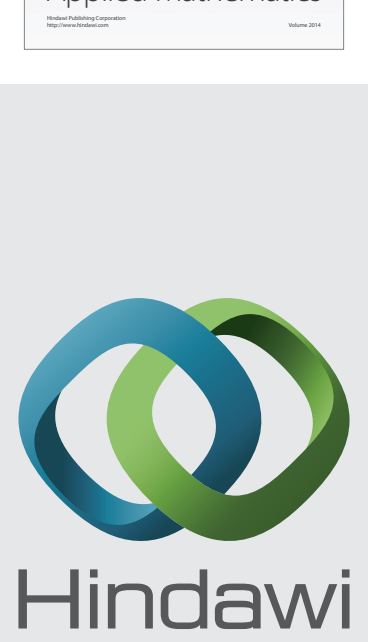

Submit your manuscripts at http://www.hindawi.com
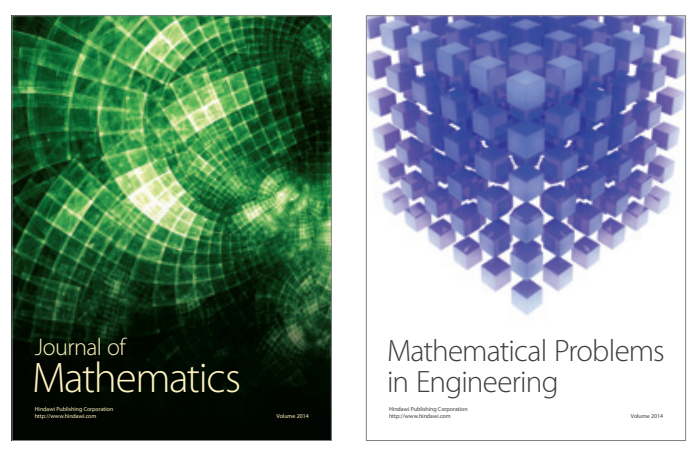

Mathematical Problems in Engineering
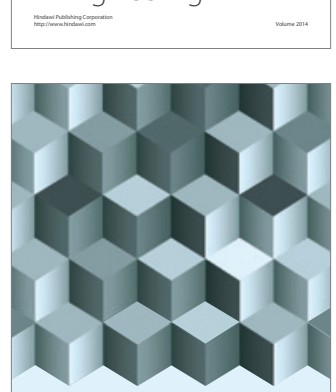

Journal of

Function Spaces
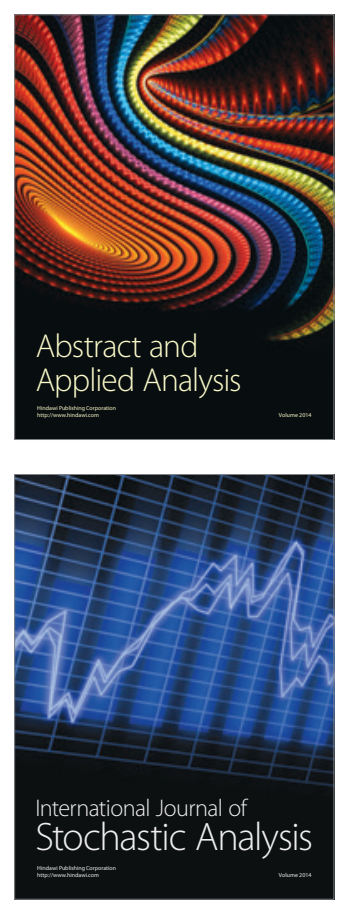

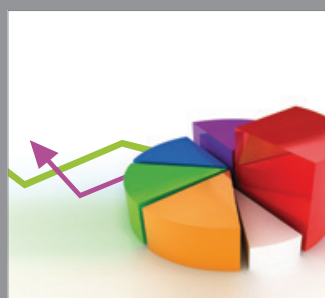

ournal of

Probability and Statistics

Promensencen
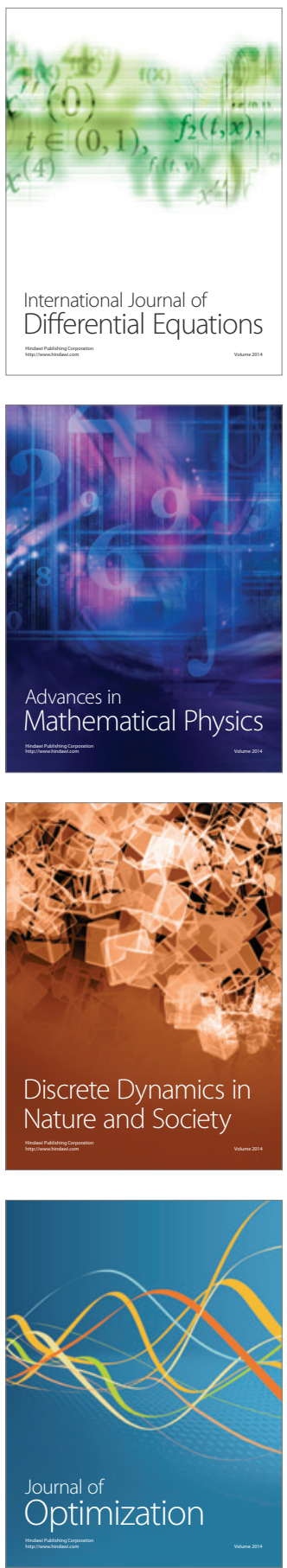